\title{
Impact of repeated clopidogrel reloading doses and increased maintenance dose in patients with acute coronary syndrome and persistent low response to clopidogrel in 30-day follow-up
}

\author{
Miroslav Krpan, Jure Samardžić1, Marija Brestovac², Boško Skorić1, Hrvoje Jurin', Vedran
} Velagić1, Davor Miličićc ${ }^{1}$

${ }^{1}$ University of Zagreb School of Medicine, University Hospital Centre Zagreb, Zagreb, Croatia

${ }^{2}$ Dr. Andrija Štampar Institute of Public Health, Zagreb, Croatia

Objectives: Platelet response to clopidogrel is not uniform. It is known that inadequate effect of clopidogrel on platelets increases the risk for new ischeamic events ${ }^{2-3}$. We aimed to investigate whether repeated clopidogrel reloading regimen and increased maintenance dose in persistent low responders is an effective and a safe strategy.

Patients and Methods: Patients with acute coronary syndrome who underwent coronary stenting were tested with impendance aggregometer - Multiplate ${ }^{\circledR}$ function analyzer. This is a point of care instrument which measures residual platelet reactivity (RPR) in small quantities of the whole blood. Low clopidogrel responders received another loading dose and underwent aggregometry testing the following day. The remaining low responders on repeated aggregometry received the third loading dose followed by a maintenance dose of $150 \mathrm{mg} /$ day. On day 7 , we repeated aggregometry testing and patients who remained low responders were assigned to a clopidogrel maintenance dose of $300 \mathrm{mg} /$ day. After one month follow-up we conducted control interview and Multiplate ${ }^{\circledR}$ testing.

Results: Persistent low response to clopidogrel was found in 12 patients out of 43 initially low responders (27.9\%). After one month of follow-up no patient had a major bleeding event. Furthermore, three patients sustained a new ischaemic event: non fatal myocardial infarction, ischaemic (fatal) stroke and in-stent thrombosis. After 30 days, seven patients (63.6\%) still had high RPR.

Conclusion: Our study showed that repeated clopidogrel reloading doses and very high maintenance doses can be safe in persistent low responders in terms of bleeding complications. However, certain number of patients are still inadequately treated and sustain new ischaemic events. Changing clopidogrel by a new thienopyridine like ticagrelor or prasugrel should be considered for those patients, if available.

KEYWORDS: acute coronary syndrome, clopidogrel, residual platelet reactivity.

CITATION: Cardiol Croat. 2013;8(9):280.

Received: $28^{\text {th }}$ Jul 2013

*Address for correspondence: Klinički bolnički centar Zagreb, Kišpatićeva 12 HR-10000 Zagreb, Croatia.

Phone: +385-1-2367-506

E-mail: miroslav.krpan@gmail.com

Table 1. Demographic data.

\begin{tabular}{|c|c|c|c|c|c|c|c|c|c|c|c|c|}
\hline Patient & Gender & Age & ACS & HLP & HA & DM2 & Smoking & Aspirin resistance & Multivessel disease & CYP2C19*2 & MACCE & HRPR after 30 days \\
\hline 1 & $F$ & 64 & STEMI & yes & yes & no & no & yes & yes & no & no & yes \\
\hline 2 & $F$ & 81 & STEMI & yes & no & yes & no & no & no & no & no & yes \\
\hline 3 & $F$ & 45 & STEMI & yes & yes & yes & no & no & no & yes & no & yes \\
\hline 4 & $F$ & 57 & STEMI & yes & yes & yes & no & yes & yes & no & no & yes \\
\hline 5 & $M$ & 63 & STEMI & yes & yes & yes & yes & yes & no & no & isch. CVI, death & N/A \\
\hline 6 & $M$ & 57 & NSTEMI & no & no & no & no & no & yes & yes & no & yes \\
\hline 7 & $M$ & 54 & STEMI & yes & yes & yes & yes & no & yes & yes & non fatal MI & no \\
\hline 8 & $M$ & 60 & \begin{tabular}{|l|} 
NSTEMI \\
\end{tabular} & yes & yes & no & no & no & yes & no & no & no \\
\hline 9 & $M$ & 60 & NSTEMI & yes & yes & yes & no & no & yes & no & no & no \\
\hline 10 & $F$ & 68 & STEMI & yes & yes & no & yes & no & yes & no & no & yes \\
\hline 11 & $M$ & 52 & STEMI & yes & yes & no & yes & yes & no & yes & IST & yes \\
\hline 12 & $\mathrm{~F}$ & 70 & STEMI & yes & yes & yes & no & no & yes & no & no & no \\
\hline
\end{tabular}

$\mathrm{HLP}=$ hyperlipidemia, $\mathrm{HA}$ = arterial hypertension, $\mathrm{DM} 2$ = diabetes mellitus type $2, \mathrm{MACCE}$ = major adverse cardiac and cerebrovascular event, $\mathrm{CVI}=$ stroke, $\mathrm{Ml}=$ myocardial infarction, HRPR $=$ high residual platelet reactivity.

\section{Literature}

1. Angiolillo DJ, Fernandez-Ortiz A, Bernardo E, et al. Variability in individual responsiveness to clopidogrel: clinical implications, management, and future perspectives. J Am Coll Cardiol. 2007;49(14):1505-16.

2. Wenaweser P, Hess O. Stent thrombosis is associated with an impaired response to antiplatelet therapy. J Am Coll Cardiol. 2005;46(5):CS5-6.

3. Price MJ, Endemann S, Gollapudi RR, et al. Prognostic significance of post-clopidogrel platelet reactivity assessed by a point-of-care assay on thrombotic events after drug-eluting stent implantation. Eur Heart J. 2008;29(8):992-1000. 